\title{
Numerical investigation of influence of partially-spanning joint on the uniaxial compressive strength of rock
}

\author{
Fengshan $\mathrm{Han}^{1, a}$, Xinli $\mathrm{Wu}^{2 \mathrm{~b}}$ \\ ${ }^{1}$ Research Center for Numerical Tests on Material Failure, Dalian University, Dalian, 116622, China \\ ${ }^{2}$ College of Engineering, the Pennsylvania State University, Pennsylvania, USA \\ ahanfs@sohu.com bxinli@psu.edu \\ Corresponding author: hanfs@sohu.com
}

\begin{abstract}
Keywords: Partially spanning joint, rock, compressive strength, numerical investigation
\end{abstract}
\begin{abstract}
In this paper failure process of rock with a partially-spanning joint is presented by numerical simulation based on RFPA $^{2 \mathrm{D}}$. numerical simulation shown that mechanical behavior of jointed rock mass is governed by both the mechanical characteristics of the discontinuities and those of the intact rock, jointed rock mass behavior tends to be dominated by the mechanical properties of the weaker join planes that occur within the rock mass, rather than the mechanical properties of the intact rock,. the failure of rock with a partially-spanning joint begin first in joint, with the increase of the vertical load, joint is expanded along the joint plane within the rock mass, the damage area expands unceasingly, Numerical simulation shown that failure model of rock with a partially-spanning join, Peak load, compressive strength are near to the real physical experimental results containing partially-spanning joint.
\end{abstract}

\section{Introduction}

In the study of rock mass mechanics, crack propagation and the polymerization is an important content in the research of rock fracture mechanics. Jointed rock masses comprise intact rock pieces interspaced with discontinuities (e.g. joints, fissures, faults, cleavage, bedding planes). The overall mechanical behavior of a jointed rock mass is governed by both the mechanical characteristics of the discontinuities and those of the intact rock bridges. In rocks with intact rock strength, jointed rock mass behavior tends to be dominated by the mechanical properties of the weaker join planes that occur within the rock mass, rather than the mechanical properties of the intact rock bridges. In these cases, the geometry of joints (i.e. location, orientation, roughness, etc.) can heavily influence the engineering behavior of the jointed rock mass. Many studies [1-5] have considered the influence of joint geometry on the overall mechanical behavior of jointed rocks, the vast majority of which have involved testing on synthetic materials with artificially embedded joints. These studies have produced a number of important results regarding the influence of joint orientation, trace length and degree of interconnectivity on the mechanical behavior of jointed rock masses, With the development of numerical simulation technology, Using numerical simulation method to study contains natural joint rock fracture process under outside load has become possible. RFPA, rock failure process analysis system provides the economy method to research rupture process containing natural joint rock.

\section{Numerical Model}

Based on References[1], the physical experimental programme was designed to investigate the influence of the location, orientation and trace length of partially spanning joints on the mechanical behaviour of rock-like materials loaded under uniaxial compression. Cement mortar was chosen for the experimental work. Sample preparation: Ordinary Portland Cement (OPC) and 'glass grade' sand were used to produce the cement mortar samples. The cement mortar samples were produced by mixing of OPC, sand and water at the ratio 1:3:0.7, respectively, by volume. As is shown in Fig 1. 

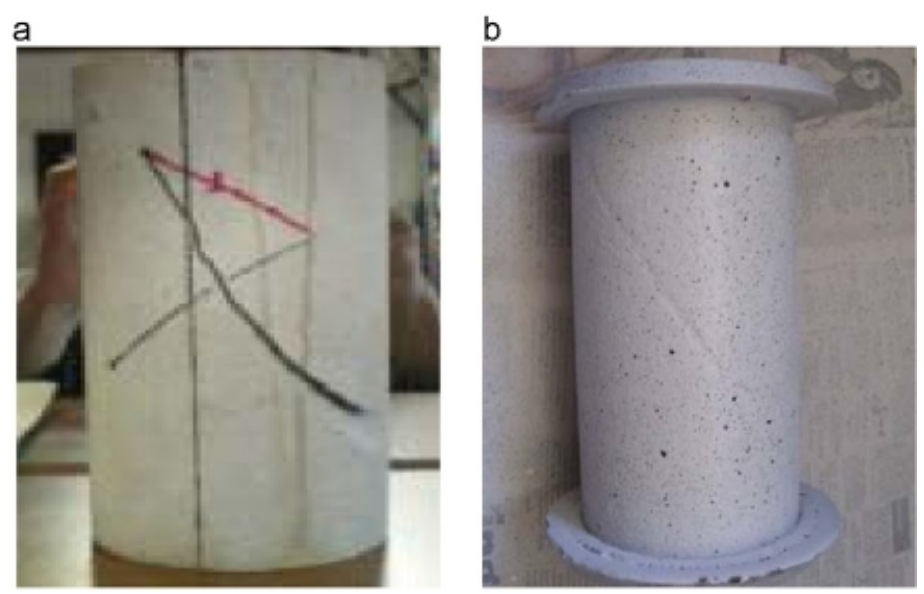

Fig.1 Model containing Partially-spanning joint for rock [1]

In order to simulate the Partially-spanning joint on rock failure process, the mesh elements used for our numerical simulation modeling is $168 \times 84$ in elements with a geometry of $\varphi 42 \mathrm{~mm} \times 168 \mathrm{~mm}$ in size, as shown in Fig. 2 and the simulated rock sample for sand is with a geometry of $\varphi 42 \mathrm{~mm} \times 168 \mathrm{~mm}$ in size, all the elements have the same size, the mechanical parameters, such as strength and elastic modulus of element, according to the statistical strength theory obeys weibull distribution

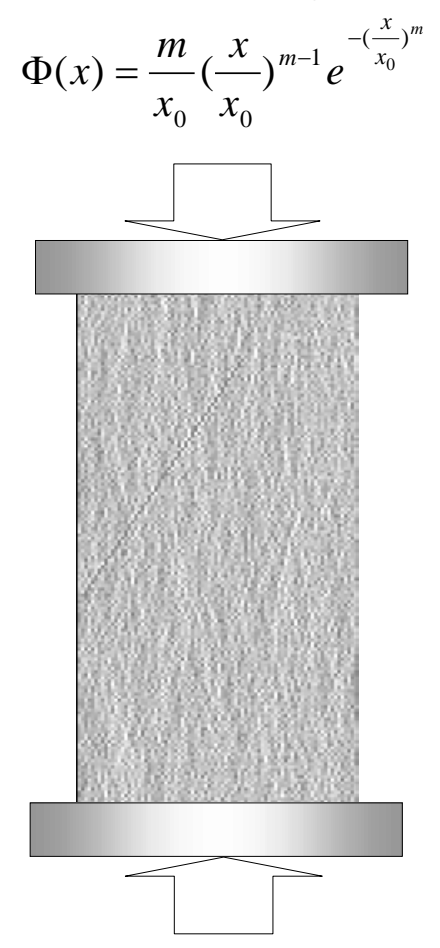

Fig.2 Numerical simulation model containing Partially-spanning joint for rock

\section{Numerical simulation parameters}

Numerical simulation model of the sandstone rock mechanics parameters are shown in Table 1, the numerical model of the joint mechanical parameters are shown in Table 2. 
Table 1.Parameters of numerical simulation for sandstone

Homogeneneity index, m 3

Mean elastic modulus, $E_{\circ}$, Gpa 50

Average compressive strength of elements for rock $\sigma_{o}$, Mpa 100

Internal friction angle, $\phi^{\circ}$

Poisson's ratio,

$\mu$

0.25

Ratio of compressive to tensile strength, $\mathrm{C} / \mathrm{T}$

10

Table 2. Parameters of numerical simulation for partially-spanning joint

$\begin{array}{lc}\text { Homogeneneity index, m } & 2 \\ \text { Mean elastic modulus, } E_{\circ} \text {, Gpa } & 5 \\ \text { Average compressive strength of elements, Mpa } & 30 \\ \text { Internal friction angle, } \phi^{\circ} & 38 \\ \text { Poisson's ratio, } \quad \mu & 0.25\end{array}$

Boundary conditions and loading method for Numerical simulation model of axial pressure load by displacement control, the initial value of axial load displacement is $0.001 \mathrm{~mm}$, axial load displacement increment is $0.001 \mathrm{~mm} /$ each step, the total number of numerical simulation is 500 steps.

\section{. Numerical results}

Based on RFPA ${ }^{2 D}$. Numerical simulation of failure process containing partially-spanning join is shown in figure 3. numerical simulation curve of peak load is shown in figure 4 . Numerical results shown that contains a single containing partially-spanning join sandstone sample, peak load Nmax $=409767(\mathrm{~N})$. Therefore, Compressive strength of sand rock containing partially-spanning join,is 7.4 Mpa, That is near to the real physical experimente results containing partially-spanning join sandstone sample, However, Based on References[1], the real physical experimente results containing partially-spanning join sandstone sample is shown in figure 5 , the real physical experimente results containing partially-spanning join, Compressive strength of rock is (9--10) Mpa

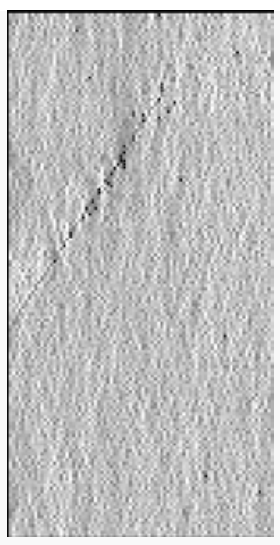

Step 75

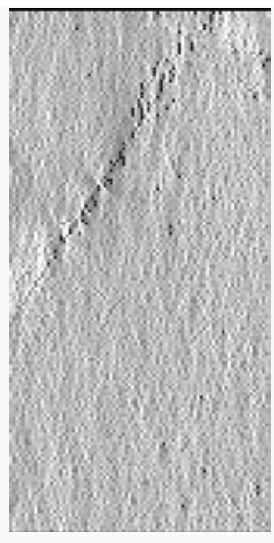

Step 98

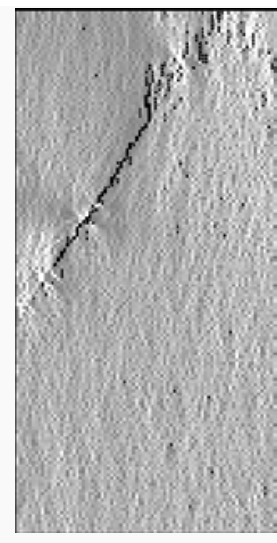

Step 105

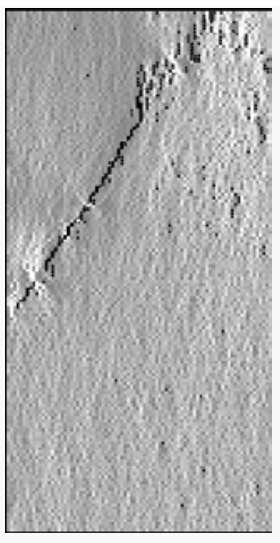

Step 110

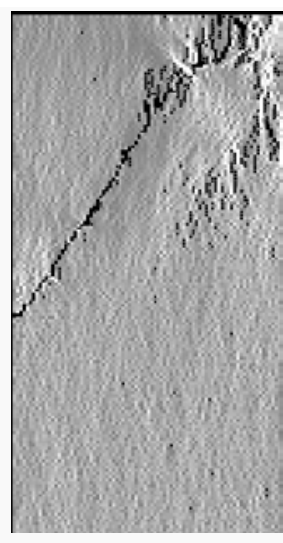

Step 123

Fig 3. Failure process of compressive experiment of rock mass with a partially-spanning join by numerical simulation. 


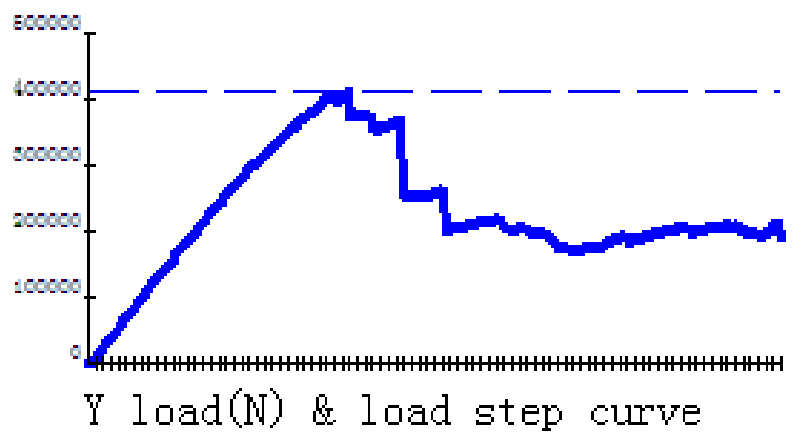

Fig4. The curve of peak load for Numerical simulation

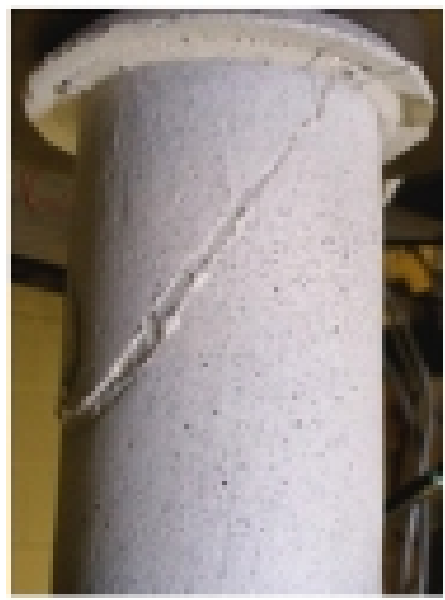

Fig5.The real physical experimente resulte containing partially-spanning join sand sample[1]

\section{Acknowledgements}

This research is funded by the "Project of science and technical program of the Educational Department of Liaoning Province (L2012436)"

\section{References}

[1] P.L.P. Wasantha, P.G. Ranjith n, D.R. Viete, L. Luo,Influence of the geometry of partially-spanning joints on the uniaxial compressive strength of rock, International Journal of Rock Mechanics \& Mining Sciences[J], 2012, 50:140-146.

[2] C.A.Tang, Numerical simulation of progressive rock falure and associated seismicity. Int J Rock Mech Min Sci[J] 1997.34(2):249-261

[3] C.A.Tang, S.Q.Kou, Crack propagation and coalescence in brittle material under compression, Engineering Fracture Mechanics [J]. 1998.61:311-324

[4] C.A.Tang, Numerical simulation of progressive rock falure and associated seismicity. Int J Rock Mech Min Sci[J] 1997.34(2):249-261

[5] C.A.Tang, Numerical test on micro-macro relationship of rock failure under uniaxial compresion ,part I: effect of heterogeneneity, Int J Rock Mech Min Sci[J],2000, 37: 555-569

[6] C.A.Tang,.B.Zhang,Z.Z.Liang,T.Xu,L.G.Tham,P_A.Lindqvist,S.Q.Kou,H.Y.Liu,Fracture spacing in layered materials and polygonal fracture, Physical Review E [J], 2006, 73(3): 70-83 